Chin. Sci. Bulletin, 1999, 44(23), 2465-2470 (Chinese Ed.); 2000, 45:9 (English Ed.), 769-774

\title{
Eigenvalues, inequalities and ergodic theory
}

\author{
$\mathrm{Mu}-\mathrm{Fa}$ Chen \\ (Beijing Normal University, Beijing 100875)
}

\begin{abstract}
This paper surveys the main results obtained during the period 1992-1999 on three aspects mentioned at the title. The first result is a new and general variational formula for the lower bound of spectral gap (i.e., the first non-trivial eigenvalue) of elliptic operators in Euclidean space, Laplacian on Riemannian manifolds or Markov chains ( $\S 1)$. Here, a probabilistic method-coupling method is adopted. The new formula is a dual of the classical variational formula. The last formula is actually equivalent to Poincaré inequality. To which, there are closely related logarithmic Sobolev inequality, Nash inequality, Liggett inequality and so on. These inequalities are treated in a unified way by using Cheeger's method which comes from Riemannian geometry. This consists of $\S 2$. The results on these two aspects are mainly completed by the author joint with $F$. Y. Wang. Furthermore, a diagram of the inequalities and the traditional three types of ergodicity is presented $(\S 3)$. The diagram extends the ergodic theory of Markov processes. The details of the methods used in the paper will be explained in a subsequent paper under the same title.
\end{abstract}

Keywords Eigenvalue inequality ergodic theory Markov process

\section{New variational formula for the lower bound of spectral gap}

\subsection{Story of estimating $\lambda_{1}$ in geometry}

We recall the study on $\lambda_{1}$ in geometry. From the story below, one should have some feeling about the difficulty of the hard mathematical topic.

Consider Laplacian $\Delta$ on a compact Riemannian manifold $(M, g)$, where $g$ is the Riemannian metric. The spectrum of $\Delta$ is discrete: $\cdots \leqslant-\lambda_{2} \leqslant-\lambda_{1}<-\lambda_{0}=0$ (may be repeated). Estimating these eigenvalues $\lambda_{k}$ (especially $\lambda_{1}$ ) consists an important section and chapter of the modern geometry. As far as we know, until now, five books have been devoted to this topic. Here we list only the geometric books but ignore the ones on general spectral theory ${ }^{[1]-[5]}$. Denote by $d, D$ and $K$ respectively the dimension, the diameter and the lower bound of Ricci curvature ( $\operatorname{Ricci}_{M} \geqslant K g$ ) of the manifold $M$. We are interested in estimating $\lambda_{1}$ in terms of these three geometric quantities. For an upper bound, it is relatively easy. Applying a test function $f \in C^{1}(M)$ to the classical variational formula

$$
\lambda_{1}=\inf \left\{\int_{M}\|\nabla f\|^{2}: f \in C^{1}(M), \int f \mathrm{~d} x=0, \int f^{2} \mathrm{~d} x=1\right\},
$$

where " $\mathrm{d} x$ " is the Riemannian volume element, one gets an upper bound. However, the lower bound is much harder. The previous works have studied the lower estimates case by case by using different 
elegant methods. Eight of the most beautiful lower bounds are listed in the following table.

$$
\begin{aligned}
& \text { A. Lichnerowicz (1958) } \\
& \text { P. H. Bérard, G. Besson } \\
& \text { \& S. Gallot (1985) } \\
& \text { P. Li \& S. T. Yau (1980) } \\
& \text { J. Q. Zhong \& H. C. Yang (1984) } \\
& \text { P. Li \& S. T. Yau (1980) } \\
& \text { K. R. Cai (1991) } \\
& \text { H. C. Yang (1989) \& F. Jia (1991) } \\
& \text { H. C. Yang (1989) \& F. Jia (1991) } \\
& \frac{d}{d-1} K, \quad K \geqslant 0 . \\
& d\left\{\frac{\int_{0}^{\pi / 2} \cos ^{d-1} t \mathrm{~d} t}{\int_{0}^{D / 2} \cos ^{d-1} t \mathrm{~d} t}\right\}^{2 / d}, \quad K=d-1>0 . \\
& \frac{\pi^{2}}{2 D^{2}}, \quad K \geqslant 0 \text {. } \\
& \frac{\pi^{2}}{D^{2}}, \quad K \geqslant 0 \text {. } \\
& \frac{1}{D^{2}(d-1) \exp \left[1+\sqrt{1+16 \alpha^{2}}\right]}, \quad K \leqslant 0 . \\
& \frac{\pi^{2}}{D^{2}}+K, \quad K \leqslant 0 \text {. } \\
& \frac{\pi^{2}}{D^{2}} e^{-\alpha}, \quad \text { if } d \geqslant 5, \quad K \leqslant 0 . \\
& \frac{\pi^{2}}{2 D^{2}} e^{-\alpha^{\prime}}, \quad \text { if } 2 \leqslant d \leqslant 4, \quad K \leqslant 0,
\end{aligned}
$$

where $\alpha=D \sqrt{|K|(d-1)} / 2, \quad \alpha^{\prime}=D \sqrt{|K|((d-1) \vee 2)} / 2$. All together, there are five sharp estimates $((1),(2),(4),(6)$ and $(7))$. The first two are sharp for the unit sphere in two- or higherdimension but it fails for the unit circle; the fourth, the sixth and the seventh estimates are all sharp for the unit circle. The above authors include several famous geometers and the estimates were awarded several times. From the table, it follows that the picture is now very complete, due to the effort by the geometers in the past 40 years. For such a well-developed field, what can we do now? Our original starting point is to learn from the geometers, study their methods, especially the recent new developments. It is surprising that we actually went to the opposite direction, that is, studying the first eigenvalue by using a probabilistic method. It was indeed not dreamed that we could finally find a general formula.

\subsection{New variational formula}

To state the result, we need two notations

$$
\begin{aligned}
& C(r)=\cosh ^{d-1}\left[\frac{r}{2} \sqrt{\frac{-K}{d-1}}\right], \quad r \in(0, D) . \\
& \mathcal{F}=\{f \in C[0, D]: f>0 \text { on }(0, D)\} .
\end{aligned}
$$

Here the dimension $d$, the diameter $D$ and the lower bound of Ricci curvature $K$ have all been used.

Theorem [General formula] (Chen \& Wang $\left.{ }^{[6]}\right) . \lambda_{1} \geqslant \sup _{f \in \mathcal{F}} \inf _{r \in(0, D)} \frac{4 f(r)}{\int_{0}^{r} C(s)^{-1} \mathrm{~d} s \int_{s}^{D} C(u) f(u) \mathrm{d} u}$.

The new variational formula has its essential value in estimating the lower bound. It is a dual of the classical variational formula in the sense that "inf" is replaced by "sup". The last formula goes back to Lord S. J. W. Rayleigh(1877) or E. Fischer (1905). Noticing that there are no common points in these two formulas, this explains the reason why such a formula never appeared before. Certainly, the new formula can produce a lot of new lower bounds. For instance, the one corresponding to the trivial function $f \mathcal{E} u i v 1$ is still non-trivial in geometry. Next, let $\alpha$ be the same as above and let $\beta=\frac{\pi}{2 D}$. Applying the formula to the test functions $\sin (\beta r), \sin (\alpha r), \sin (\beta r)$ and $\cosh ^{d-1}(\alpha r) \sin (\beta r)$ successively, we obtain the following: 
Corollary (Chen \& Wang $\left.{ }^{[6]}\right)$.

$$
\begin{aligned}
& \lambda_{1} \geqslant \frac{\pi^{2}}{D^{2}}+\max \left\{\frac{\pi}{4 d}, 1-\frac{2}{\pi}\right\} K, \quad K \geqslant 0 \\
& \lambda_{1} \geqslant \frac{d K}{d-1}\left\{1-\cos ^{d}\left[\frac{D}{2} \sqrt{\frac{K}{d-1}}\right]\right\}^{-1}, \quad d>1, \quad K \geqslant 0 \\
& \lambda_{1} \geqslant \frac{\pi^{2}}{D^{2}}+\left(\frac{\pi}{2}-1\right) K, \quad K \leqslant 0 \\
& \lambda_{1} \geqslant \frac{\pi^{2}}{D^{2}} \sqrt{1-\frac{2 D^{2} K}{\pi^{4}}} \cosh ^{1-d}\left[\frac{D}{2} \sqrt{\frac{-K}{d-1}}\right] \quad d>1, \quad K \leqslant 0 .
\end{aligned}
$$

\section{Comments.}

(1) The corollary improves all the estimates (1) - (8). (9) improves (4); (10) improves (1) and (2); (11) improves (6); (12) improves (7) and (8).

(2) The theorem and corollary valid also for the manifolds with convex boundary with Neumann boundary condition. In this case, the estimates (1)-(8) are believed by geometers to be true. However, only the Lichnerowicz's estimate (1) was proved by J. F. Escobar until 1990. Except this, the others in (2) - (8) (and furthermore (9) - (12)) are all new in geometry ${ }^{[6]}$.

(3) For more general non-compact manifolds, elliptic operators or Markov chains, we also have the corresponding dual variational formula ${ }^{[7],[8]}$. The point is that only three parameters $d$ , $D$ and $K$ are used in the geometric case, but there are infinite parameters in the case of elliptic operators or Markov chains. Thus, the latter cases are more complicated. Actually, the above formula is a particular example of our general formula for elliptic operators. In dimensional one, our formula is complete.

(4) The probabilistic method - coupling method was developed by the present author before this work for more than ten years. The above study was the first time for applying the method to estimating the eigenvalues. For a long time, almost nobody believes that the method can achieve sharp estimate. From these facts, the influence of the above results to probability theory and spectral theory should be clear ${ }^{[8]}$.

\section{Basic inequalities and new forms of Cheeger's constants}

\subsection{Basic inequalities}

Let $(E, \mathcal{E}, \pi)$ be a probability space satisfying $\{(x, x): x \in E\} \in \mathcal{E} \times \mathcal{E}$. Denote by $L^{p}(\pi)$ the usual real $L^{p}$-space with norm $\|\cdot\|_{p}$. Write $\|\cdot\|=\|\cdot\|_{2}$. Our main object is a symmetric form $(D, \mathcal{D}(D))$ on $L^{2}(\pi)$. For Laplacian on manifold, the form used in the last part is the following

$$
D(f):=D(f, f)=\int_{M}\|\nabla f\|^{2} \mathrm{~d} x, \quad \mathcal{D}(D) \supset C^{\infty}(M) .
$$

Here, only the diagonal elements $D(f)$ is written, but the non-diagonal elements can be then deduced from the diagonal ones by using the quadrilateral role. The classical variational formula for spectral gap now can be rewritten into the following form.

$$
\text { Poincaré inequality: } \quad \operatorname{Var}(f) \leqslant C D(f), \quad f \in L^{2}(\pi)
$$

where $\operatorname{Var}(f)=\pi\left(f^{2}\right)-\pi(f)^{2}, \pi(f)=\int f \mathrm{~d} \pi$ and $C\left(=\lambda_{1}^{-1}\right)$ is a constant. Thus, the study on the spectral gap is the same as the one on Poincaré inequality of the form $(D, \mathcal{D}(D))$. Nevertheless, we have more symmetric forms. For an elliptic operator in $\mathbb{R}^{d}$, the corresponding form is as follows.

$$
D(f)=\frac{1}{2} \int_{\mathbb{R}^{d}}\langle a(x) \nabla f(x), \nabla f(x)\rangle \pi(\mathrm{d} x), \quad \mathcal{D}(D) \supset C_{0}^{\infty}\left(\mathbb{R}^{d}\right),
$$

where $\langle\cdot, \cdot\rangle$ denotes the standard inner product in $\mathbb{R}^{d}$ and $a(x)$ is positive definite. Corresponding to an integral operator (or symmetric kernel) on $(E, \mathcal{E})$, we have the symmetric form

$$
D(f)=\frac{1}{2} \int_{E \times E} J(\mathrm{~d} x, \mathrm{~d} y)[f(y)-f(x)]^{2}, \quad \mathcal{D}(D)=\left\{f \in L^{2}(\pi): D(f)<\infty\right\},
$$


where $J$ is a non-negative, symmetric measure having no charge on the diagonal set $\{(x, x): x \in E\}$. A typical example in our mind is the reversible jump process with $q$-pair $(q(x), q(x, \mathrm{~d} y))$ and reversible measure $\pi$. Then $J(\mathrm{~d} x, \mathrm{~d} y)=\pi(\mathrm{d} x) q(x, \mathrm{~d} y)$. More especially, for a reversible $Q$-matrix $Q=\left(q_{i j}\right)$ with reversible measure $\left(\pi_{i}>0\right)$, we have density $J_{i j}=\pi_{i} q_{i j}(j \neq i)$ with respect to the counting measure.

For a given symmetric form $(D, \mathcal{D}(D))$, except Poincaré inequality, there are also other basic inequalities.

$$
\begin{array}{llrl}
\text { Nash inequality: } & \operatorname{Var}(f) \leqslant C D(f)^{1 / p}\|f\|_{1}^{2 / q}, & f \in L^{2}(\pi) \\
\text { Liggett inequality: } & \operatorname{Var}(f) \leqslant C D(f)^{1 / p} \operatorname{Lip}(f)^{2 / q}, \quad f \in L^{2}(\pi)
\end{array}
$$

where $C$ is a constant and $\operatorname{Lip}(f)$ is the Lipschitz constant of $f$ with respect to some distance $\rho$. The above three inequalities are actually particular cases of the following one

$$
\text { Liggett-Stroock inequality: } \quad \operatorname{Var}(f) \leqslant C D(f)^{1 / p} V(f)^{1 / q}, \quad f \in L^{2}(\pi)
$$

where $V: L^{2}(\pi) \rightarrow[0, \infty]$ is homogeneous of degree two: $V\left(c_{1} f+c_{2}\right)=c_{1}^{2} V(F), c_{1}, c_{2} \in \mathbb{R}$. Another closely related one is

$$
\text { Logarithmic Sobolev inequality: } \quad \int f^{2} \log \left(f^{2} / \mid f \|^{2}\right) \mathrm{d} \pi \leqslant C D(f), \quad f \in L^{2}(\pi) \text {. }
$$

\subsection{Statue of the research}

From now on, we restrict ourselves to the symmetric form (13) corresponding to integral operators. The question is under what condition on the symmetric measure $J$, the above inequalities hold. In contrast with the probabilistic method used in the last part, here we adopt Cheeger's method (1970) which comes from Riemannian geometry.

We call $\lambda_{1}:=\inf \{D(f): \pi(f)=0,\|f\|=1\}$ the spectral gap of the form $(D, \mathcal{D}(D))$. For bounded jump processes, the main known result is the following.

Theorem (Lawler \& Sokal (1988)). $\quad \lambda_{1} \geqslant \frac{k^{2}}{2 M}$, where $k=\inf _{\pi(A) \in(0,1)} \frac{\int_{A} \pi(\mathrm{d} x) q\left(x, A^{c}\right)}{\pi(A) \wedge \pi\left(A^{c}\right)}, M=$ $\sup _{x \in E} q(x)$.

In the past seven years, the theorem has been collected into six books ${ }^{[9]}$ - $[14]$. From the titles of the books, one sees the wider range of the applications of the study. The problem is: the result fails for unbounded operator. Thus, it has been a challenge open problem in the past ten years or more to handle the unbounded situation.

As for logarithmic Sobolev inequality, there is a large number of publications in the past twenty years or more for differential operators. However, there was almost no result for integral operators until the next result appeared.

Theorem (Diaconis \& Saloff-Coste (1996)). Let $E$ be a finite set and $\sum_{j}\left|q_{i j}\right|=1$ holds for all $i$. Then the logarithmic Sobolev constant $\sigma:=\inf \left\{D(f) / \int f^{2} \log [|f| /\|f\|]:\|f\|=1\right\}$ satisfies $\sigma \geqslant \frac{2\left(1-2 \pi_{*}\right) \lambda_{1}}{\log \left[1 / \pi_{*}-1\right]}$, where $\pi_{*}=\min _{i} \pi_{i}$.

Obviously, the result fails again for infinite $E$. The problem is due to the limitation of the method used in the proof.

\subsection{New result}

Corresponding to three inequalities, we introduce respectively the following new forms of Cheeger's 
constants.

$$
\begin{aligned}
& \underline{\text { Inequality }} \quad \underline{\text { Constant } k^{(\alpha)}} \\
& \text { Poincaré } \left.\quad \inf _{\pi(A) \in(0,1)} \frac{J^{(\alpha)}\left(A \times A^{c}\right)}{\pi(A) \wedge \pi\left(A^{c}\right)} \quad \text { (Chen \& Wang }{ }^{[15]}\right) \\
& \text { Nash } \quad \inf _{\pi(A) \in(0,1)} \frac{J^{(\alpha)}\left(A \times A^{c}\right)}{\left[\pi(A) \wedge \pi\left(A^{c}\right)\right]^{(\nu-1) / \nu}} \quad\left(\text { Chen }^{[16]}\right) \\
& \text { Log. Sobolev } \quad \lim _{r \rightarrow 0} \inf _{\pi(A) \in(0, r]} \frac{J^{(\alpha)}\left(A \times A^{c}\right)}{\pi(A) \sqrt{\log \left[e+\pi(A)^{-1}\right]}} \quad \text { (Wang }{ }^{[17]} \text { ) } \\
& \lim _{\delta \rightarrow \infty} \inf _{\pi(A)>0} \frac{J^{(\alpha)}\left(A \times A^{c}\right)+\delta \pi(A)}{\pi(A) \sqrt{1-\log \pi(A)}} \quad\left(\text { Chen }^{[18]}\right)
\end{aligned}
$$

where $r(x, y)$ is a symmetric, non-negative function such that $J^{(\alpha)}(\mathrm{d} x, \mathrm{~d} y):=I_{\{r(x, y)>0\}} \frac{J(\mathrm{~d} x, \mathrm{~d} y)}{r(x, y)^{\alpha}}$ $(\alpha>0)$ satisfies $\frac{J^{(1)}(\mathrm{d} x, E)}{\pi(\mathrm{d} x)} \leqslant 1, \pi$-a.s. For convenience, we use the convention $J^{(0)}=J$. Now, our main result can be easily stated as follows.

Theorem. $k^{(1 / 2)}>0 \Longrightarrow$ the corresponding inequality holds.

The result is proved in four papers [15] - [18]. At the same time, some estimates for the upper or lower bounds are also presented. These estimates can be sharp or qualitatively sharp, which did not happen before in using Cheeger's technique.

\section{New picture of ergodic theory}

\subsection{Importance of the inequalities}

Let $\left(P_{t}\right)_{t \geqslant 0}$ be the semigroup determined by the symmetric form $(D, \mathcal{D}(D))$. Then, various applications of the inequalities are based on the following result.

\section{Theorem.}

(1) Let $V\left(P_{t} f\right) \leqslant V(f)$ for all $t \geqslant 0$ and $f \in L^{2}(\pi)$ (which is automatic when $V(f)=\|f\|_{r}^{2}$ ). Then Liggett-Stroock inequality implies that

$$
\operatorname{Var}\left(P_{t} f\right) \leqslant C V(f) / t^{q-1}, \quad t>0 .
$$

(2) Conversely, (14) $\Longrightarrow$ Liggett-Stroock inequality.

(3) Poincaré inequality $\Longleftrightarrow \operatorname{Var}\left(P_{t} f\right) \leqslant \operatorname{Var}(f) \exp \left[-2 \lambda_{1} t\right]$.

Note that $\operatorname{Var}\left(P_{t} f\right)=\left\|P_{t} f-\pi(f)\right\|^{2}$. Therefore, the above inequalities describe some type of $L^{2}$-ergodicity of the semigroup $\left(P_{t}\right)_{t \geqslant 0}$. In particular, we call (14) $L^{2}$-algebraic convergence. These inequalities have become powerful tools in the study on infinite-dimensional mathematics (phase transitions, for instance) and the effectiveness of random algorithms.

\subsection{Three traditional types of ergodicity}

In the study of Markov processes, the following three types of ergodicity are well known.

$$
\begin{array}{ll}
\text { Ordinary ergodicity: } \quad & \lim _{t \rightarrow \infty}\left\|p_{t}(x, \cdot)-\pi\right\|_{\mathrm{Var}}=0 \\
\text { Exponential ergodicity }: \quad & \left\|p_{t}(x, \cdot)-\pi\right\|_{\mathrm{Var}} \leqslant C(x) e^{-\varepsilon t} \\
\text { Strong ergodicity }: & \lim _{t \rightarrow \infty} \sup _{x}\left\|p_{t}(x, \cdot)-\pi\right\|_{\mathrm{Var}}=0
\end{array}
$$

where $p_{t}(x, \mathrm{~d} y)$ is the transition function of the Markov process and $\|\cdot\|_{\text {Var }}$ is the total variation norm. They obey the following relation: Strong ergodicity $\Longrightarrow$ Exponential ergodicity $\Longrightarrow$ Ordinary ergodicity. Now, it is natural to ask the following question. Does there exist any relation between the above inequalities and the traditional three types of ergodicity? 


\subsection{New picture of ergodic theory}

Theorem $^{[16],[19],[20]}$. For reversible Markov chains, we have the following diagram:

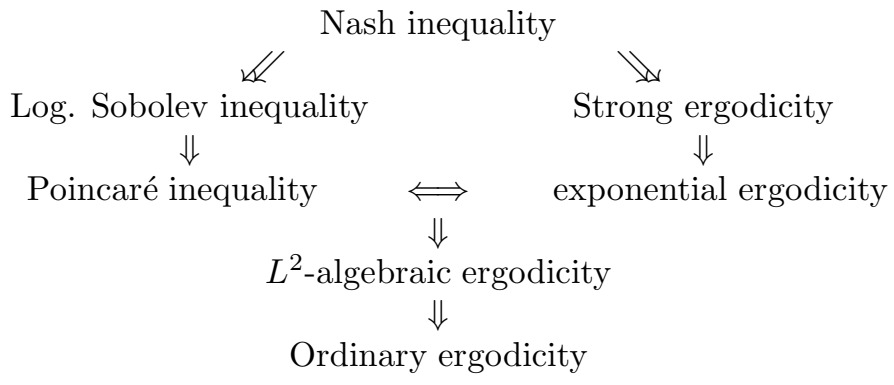

where $L^{2}$-algebraic ergodicity means that (14) holds for some $V$ having the properties: $V$ is homogeneous of degree two, $V(f)<\infty$ for all functions $f$ with finite support.

\section{Comments.}

(1) The diagram is complete in the following sense. Each single-side implication can not be replaced by double-sides one. Moreover, strong ergodicity and logarithmic Sobolev inequality are not comparable.

(2) The application of the diagram is obvious. For instance, one obtains immediately some criteria (which are indeed new) for Poincaré inequality to be held from the well-known criteria for the exponential ergodicity. On the other hand, by using the estimates obtained from the study on Poincaré inequality, one may estimate exponentially ergodic convergence rate (for which, the knowledge is still very limited).

(3) Except the equivalence, all the implications in the diagram are suitable for more general Markov processes. The equivalence in the diagram should be also suitable for more Markov processes but it may be false in the infinite-dimensional situation.

(4) No doubt, the diagram extends the ergodic theory of Markov processes.

Acknowledgement Research supported in part by NSFC (No. 19631060), Math. Tian Yuan Found., Qiu Shi Sci. \& Tech. Found., RFDP and MCME.

\section{References}

[1]. Chavel I., Eigenvalues in Riemannian Geometry, New York: Academic Press, 1984.

[2]. Bérard P H, Spectral Geometry: Direct and Inverse Problem, LNM. vol 1207, New York: Springer-Verlag, 1986.

[3]. Yau S T, Schoen R, Differential Geometry (In Chinese), Beijing: Science Press, 1988.

[4]. Li P, Lecture Notes on Geometric Analysis, Seoul National Univ, Korea, 1993.

[5]. Ma C Y, The Spectrum of Riemannian Manifolds (In Chinese), Nanjing: Press of Nanjing U, 1993.

[6]. Chen M F, Wang F Y, General formula for lower bound of the first eigenvalue, Sci Sin, 1997, 40:4, 384-394.

[7]. Chen M F, Wang F Y, Estimation of spectral gap for elliptic operators, Trans Amer Math Soc, 1997, 349: 1239-1267.

[8]. Chen M F, Coupling, spectral gap and related topics, Chin Sci Bulletin, 1997, (I): 42:16, 13211327; (II): 42:17, 1409-1416; (III): 42:18, 1497-1505.

[9]. Chen M F, From Markov Chains to Non-Equilibrium Particle Systems, Singapore: World Scientific, 1992.

[10]. Sinclair A, Algorithms for Random Generation and Counting: A Markov Chain Approach, Boston: Birkhäuser, 1993.

[11]. Saloff-Coste L, Lectures on finite Markov chains, LNM 1665, 301-413, New York: SpringerVerlag, 1997.

[12]. Chung F R K, Spectral Graph Theory, CBMS, 92, Rhode Island: AMS, Providence, 1997.

[13]. Colin de Verdière Y, Spectres de Graphes, Paris: Publ Soc Math France, 1998. 
[14]. Aldous D G \& Fill J A (1994-), Reversible Markov Chains and Random Walks on Graphs, URL www. stat.Berkeley.edu/users/aldous/book.html.

[15]. Chen M F, Wang F Y, Cheeger's inequalities for general symmetric forms and existence criteria for spectral gap, Abstract. Chin Sci Bulletin, 1998, 43:18, 1516-1519. Ann. Prob. 2000, 28:1, 235-257.

[16]. Chen M F, Nash inequalities for general symmetric forms, Acta Math Sin Eng Ser, 1999, 15:3, 353-370.

[17]. Wang F Y, Sobolev type inequalities for general symmetric forms, to appear in Proc Amer Math Soc, 1999.

[18]. Chen M F, Logarithmic Sobolev inequality for symmetric forms, Sci Chin, 2000, 43:6, 601-608.

[19]. Chen M F, Equivalence of exponential ergodicity and $L^{2}$-exponential convergence for Markov chains, Stoch Proc Appl, 2000,87, 281-297.

[20]. Chen M F, A new story of ergodic theory, to appear in Proceedings of IMS Workshop on Applied Probability, Hong Kong: Intern. Press.

(Received August 2, 1999) 